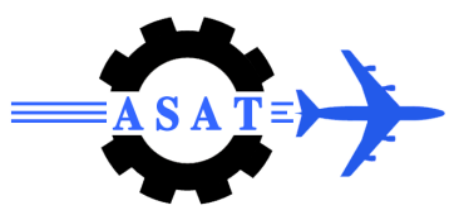

\title{
A New CFAR Processor Based on Guard Cells Information
}

\author{
\{A. Mostafa, F.M. Ahmed, K.H. Moustafa, and Y.Z. Elhalwagy\} ${ }^{*}$
}

\begin{abstract}
Radar detection procedures involve the comparison of the received signal amplitude to a threshold. In order to obtain a constant false-alarm rate (CFAR), an adaptive threshold must be applied reflecting the local clutter situation. This paper presents an intelligent CFAR technique based on comparing the performance of five existing CFAR processors at different target and clutter situations. The proposed intelligent CFAR processor selects the adaptive threshold which is calculated by the best CFAR processor for certain environmental condition. The selection criterion based on comparing the information contained in the guard cells to those contained in test and window cells. This comparison is done to differentiate between single target, multiple targets, and clutter transition situations. Performance comparison through the Receiver Operating Characteristic (ROC) is carried out to validate the superiority of the proposed CFAR technique at different target and clutter situations.
\end{abstract}

\section{Introduction}

The task of primary radars used in air or vessel traffic control is to detect all objects within the area of observation and to estimate their positional coordinates. Generally, target detection would be an easy task if the echoing objects were located in front of an otherwise clear or empty background. In such a case the echo signal can simply be compared with a fixed threshold, and targets are detected whenever the signal exceeds this threshold [1]. In real radar application, the target always appears before a background filled with point, area, or extended clutter. Frequently the location of this background clutter is additionally subject to variations in time and position. This fact calls for adaptive signal processing techniques operating with a variable detection threshold to be determined in accordance to the local clutter situation. This is achieved by the use of CFAR processors which adaptively set the detection threshold based on the background information. However, the radar receiver processing chain and the position of the CFAR detector is shown in figure 1 [2].

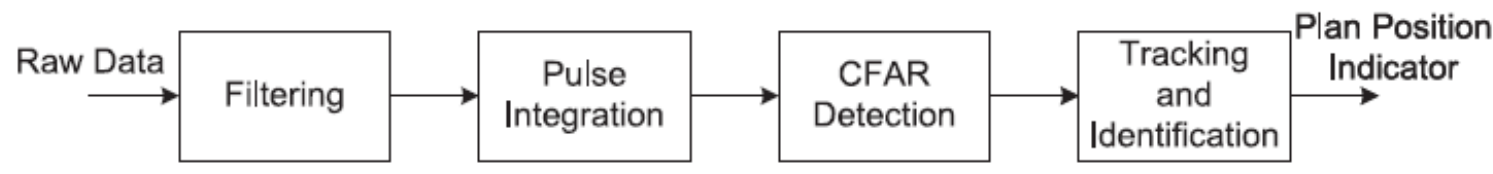

Fig. 1 Traditional Signal Processing Radar Chain.

\footnotetext{
${ }^{*}$ Egyptian Armed Forces, Egypt.
} 
A relatively simple CFAR algorithm uses the average received energy in $2 \mathrm{~N}$ nearby range cells to obtain a threshold and is called Cell Average (CA)-CFAR processor. The performance of this algorithm is degraded in case of non-homogeneous background. Different CFAR schemes were designed to overcome different problems caused by the existence of nonhomogeneous background.

Unfortunately, there is no optimum CFAR processor that can deal with all CFAR problems. However, different CFAR problems and their solutions are discussed in section 2. In this paper, an intelligent CFAR processor designated as Intelligent (INT) CFAR is proposed. The proposed INT-CFAR technique compares between the performances of different CFAR processors at different target and clutter situations and chooses the best performance for each case. By doing so, a near optimum CFAR processor can be obtained. The idea of the proposed INT-CFAR is discussed in details in section 3. Performance evaluation of the proposed INT CFAR compared to other CFAR techniques through the ROC at different target and clutter situations is introduced in section 4. Finally, the conclusion comes at section 5.

\section{CFAR Problems and Related Solutions}

Most CFAR processors cannot maintain the optimal performance because of the violation of certain assumptions about the environment. For the CA-CFAR, the inherent assumption is that the statistics of the interference at each reference cell are the same as the statistics of the test cell. There are two common situations in which this condition is not met, leading to two major problems that require careful investigation in such a CFAR detection scheme. These problems presented by the regions of clutter power transitions or edges and targets returns in the reference window.

Modification of the CA-CFAR schemes have been proposed to overcome the problems associated with non-homogeneous noise backgrounds. This scheme splits the reference window into leading and lagging parts symmetrically about the cell under test. The noise power is no longer estimated efficiently, and therefore, some loss of detection in the homogeneous reference window is introduced compared with the CA-CFAR processor. Hansen [4] has proposed a CFAR procedure to regulate false alarm rate in the region of clutter transition; in this procedure the noise power is estimated by the greatest of (GO) the sums in the leading and lagging windows. The additional loss of detection performance (in terms of signal to noise ratio) over the CA-CFAR procedure in homogeneous reference window is typically found to be between 0.1-0.3 dB [5]. Moore \& Lawrence [6] have shown that during clutter power transitions, a minor increase can be expected in the false alarm rate of the GO-CFAR processor in the worst case when the lagging window contains radar returns from the clear background while the leading window contains returns from the high clutter region. However, the GO-CFAR detector is incapable of resolving closely spaced targets. Weiss [7] has shown that detection probability decreases intolerably when a single interfering target with strength equal to that of the primary target appears in the reference window.

In order to prevent the suppression of closely spaced targets. Trunk [8] proposed a SO-CFAR processing scheme in which the smallest of (SO) the sums in the leading and lagging windows is used to estimate the noise power. It has been shown that the SO-CFAR processor performs very well in resolving two closely spaced targets. However, the detection performance of the SO-CFAR processor degrades considerably if interfering targets are located in both the leading and lagging windows. This is simply due to the fact that at least one of the two interfering targets will influence the threshold by raising its value, leading to 
masking of the primary target. Furthermore the SO-CFAR processor also fails to maintain constant false alarm rate at clutter edges.

The central operation of the CFAR processor is shown in Figure.2. As shown in this figure, the detected video range samples after the analog to digital converter are sent serially into a shift register of length $(\mathrm{M}+3)$ stages (delay line) which is divided into leading window of length $\mathrm{M} / 2$, lagging window of length $\mathrm{M} / 2$, two guard cells (GC), and the cell under test (TC). The estimated total noise plus clutter power is obtained by summing the samples in both leading and lagging windows by the summing circuits shown based on the chosen criteria (CA, GO, or SO).

Another CFAR processors exhibit a good performance in multiple target and clutter transitions situation are the order statistics cell averaging (OSCA), order statistics greatest of (OSGO) and order statistics smallest of (OSSO) CFAR processors [11]. These order statistics detectors need to perform a rank-order operation over the leading and lagging reference cells, i.e. sort the reference cells values and then select the $\mathrm{k}^{\text {th }}$ sorted value. The OSCA, OSGO and OSSO CFAR detectors perform the selection of the $\mathrm{k}^{\text {th }}$ and $\mathrm{i}^{\text {th }}$ sorted value from the leading and lagging cells respectively. Once these two values has been selected, the statistic, Z, is calculated in a similar way as the CA, GO, or the SO CFAR processors. Although the OS CFAR processor gives a good performance in non-homogeneous background, it offers the worst performance in case of homogeneous background.

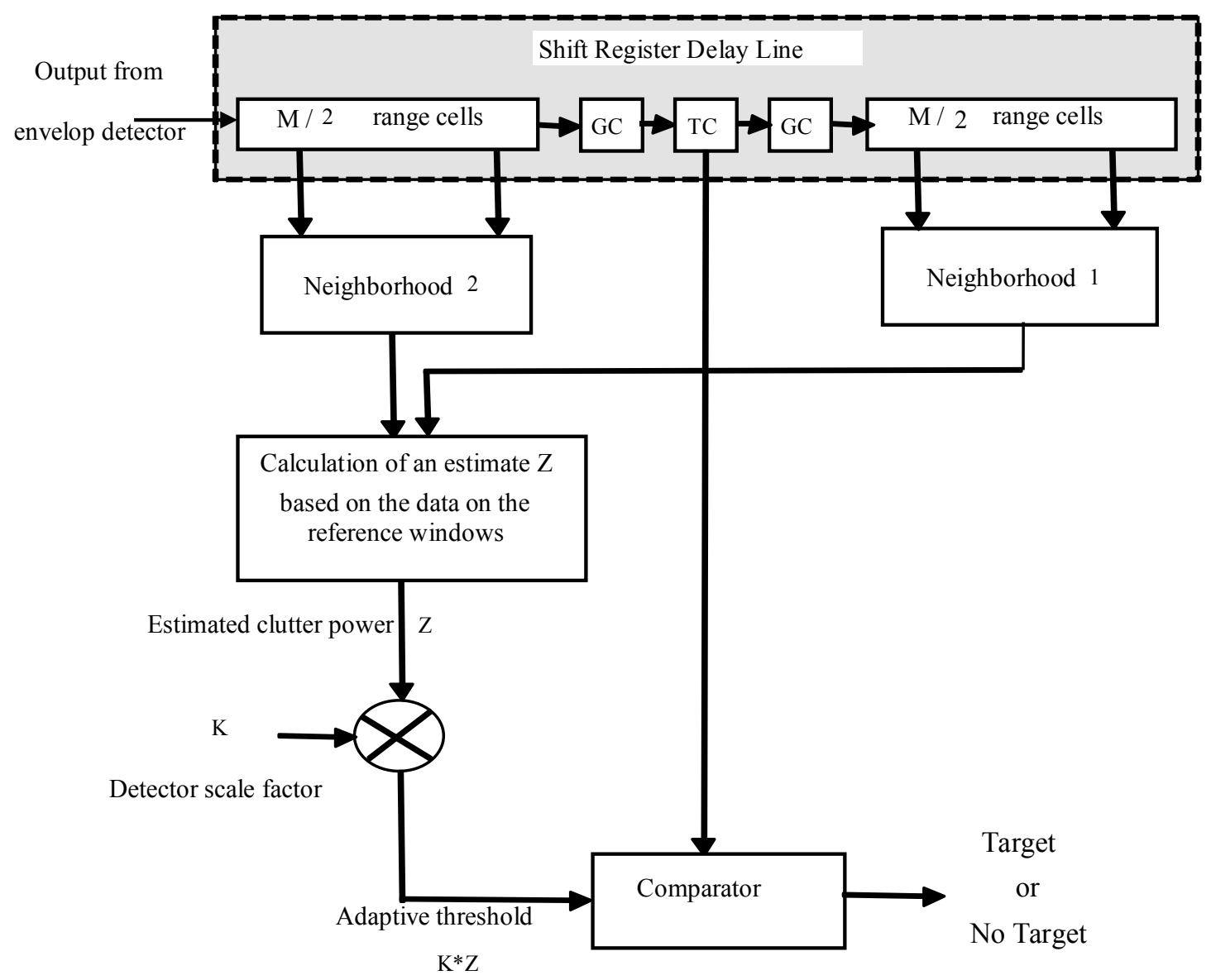

Fig. 2 The central operation of the CFAR processor 
Each one of the previously explained detectors has its own advantages and disadvantages, and may be has an optimum performance under particular environment conditions. However, the detection performance is altered by varying the number of references cells, guard cells, the CFAR detector, the k-th rank-order sample and the false alarm required [9]. For CA and GOCFAR, reference window sizes of about $\mathrm{N}=16$ to 24 are commonly used. For OS-CFAR, window sizes of about $\mathrm{N}=24$ to 32 are commonly used [1]. In this paper, a window size of $\mathrm{N}=24$ is chosen to be used in all techniques. The six previously explained CFAR detectors except the (OSSO) is considered, because its unique advantage is that it has the same processing speed as the OSGO CFAR (only half of the processing time of OS- CFAR), but it has a much higher loss than the OS CFAR and it behaves poorly in non-homogeneous clutter situations [11].

In the present work, different target and clutter situations are discussed. The Challenge is to combine the advantages of the previously discussed CFAR processors to achieve the maximum probability of detection, $\mathrm{P}_{\mathrm{d}}$, at the same time with the minimum probability of false alarm, $\mathrm{P}_{\mathrm{fa}}$.

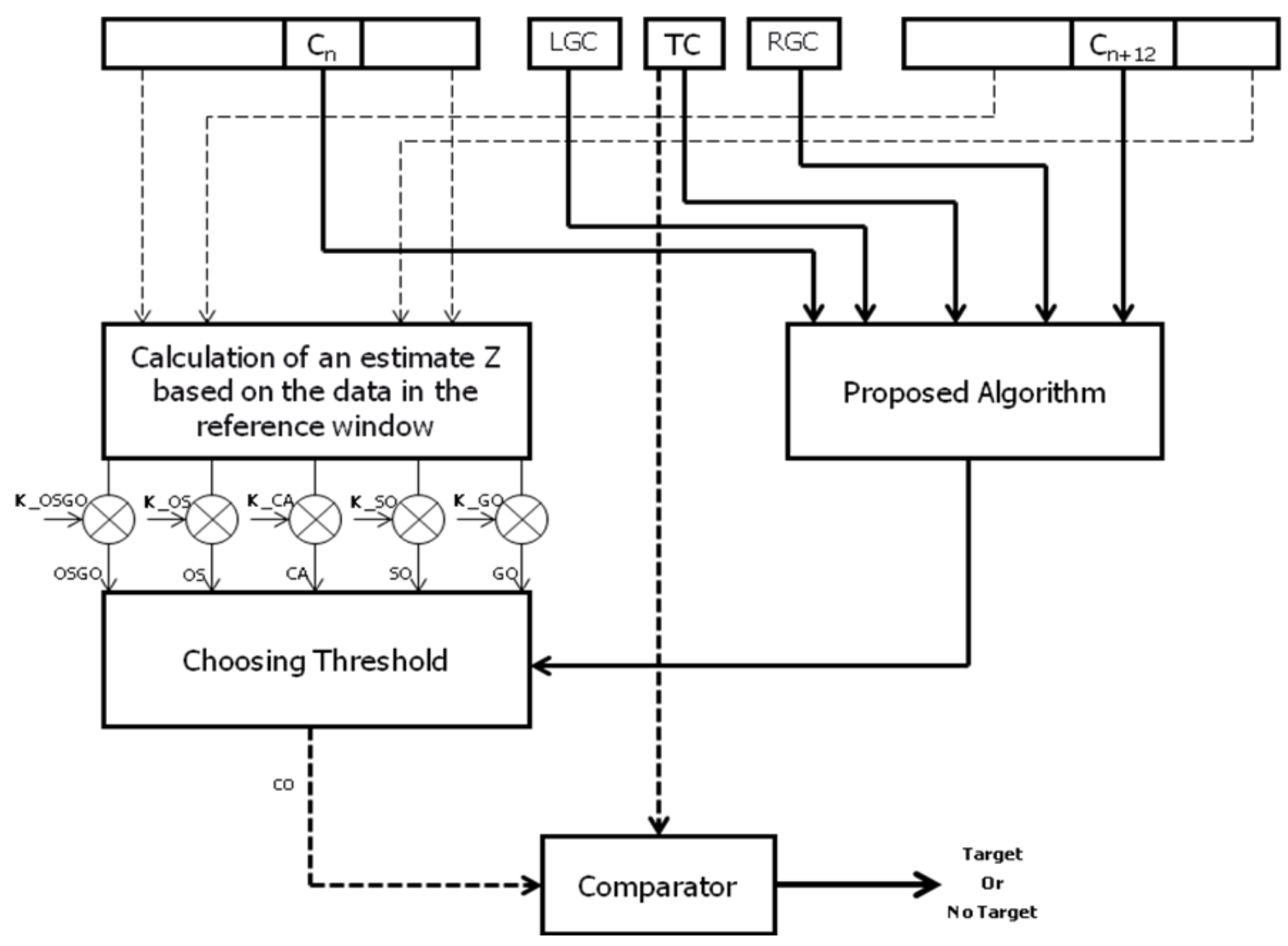

Fig. 3 Functional block diagram of the proposed INT-CFAR Processor

\section{The Proposed INT-CFAR Processor}

In this paper, an intelligent CFAR algorithm, designated as INT-CFAR, is introduced. This algorithm is intelligent enough to understand the environmental circumstances and adapt itself to introduce the optimum probability of detection, $\mathrm{P}_{\mathrm{d}}$, with the lowest probability of false alarm, $\mathrm{P}_{\mathrm{fa}}$, in all studied cases 
The function block diagram of the proposed INT-CFAR is shown in figure 3 for a chosen total window size of 24 cells. The proposed algorithm uses the information contained in the guard cells beside the test cell. Also, it uses the information contained in cells number (n) and $(\mathrm{n}+12)$ respectively $\left(\mathrm{C}_{\mathrm{n}}\right.$, and $\left.\mathrm{C}_{\mathrm{n}+12}\right)$.

The idea behind the proposed INT-CFAR is to sense the existence of a clutter transitions, based on comparing the signal amplitudes contained in both guard cells with the test cell. In this case, the threshold level obtained by the GO-CFAR is used to reduce the false alarm rate.

If this case is not met, a comparison is made between the signal summations of each window, which indicates the presence of clutter region in one of the reference windows. Also, an existence of target in the test cell is most probable. In this case, the threshold level obtained by the SO-CFAR processor is used to increase the detection probability.

A comparison is made between the signal values contained in the two guard cells and the cell number $n$ and the cell number $n+12$ to check for the clutter width. This check permits the choice of the threshold level calculated by the OS-CFAR processor which exhibits a better performance in this case.

If all the comparison leads to a homogeneous background, the value of the threshold level calculated by the CA-CFAR processor is selected.

The flowchart representing the proposed threshold selection algorithm is shown in figure 4 . All the values of the used variables; val1, val2, val3, and val4 which are used for comparison in this flowchart are obtained by simulation. The performance evaluation of the proposed INT-CFAR processor which uses this threshold selection algorithm is introduced in the following section. All target and clutter situations shall be discussed.

However, figures 5 and 6 shows different thresholds obtained from different CFAR processors and the applied criterion to select the actual threshold for the proposed INT-CFAR processor.

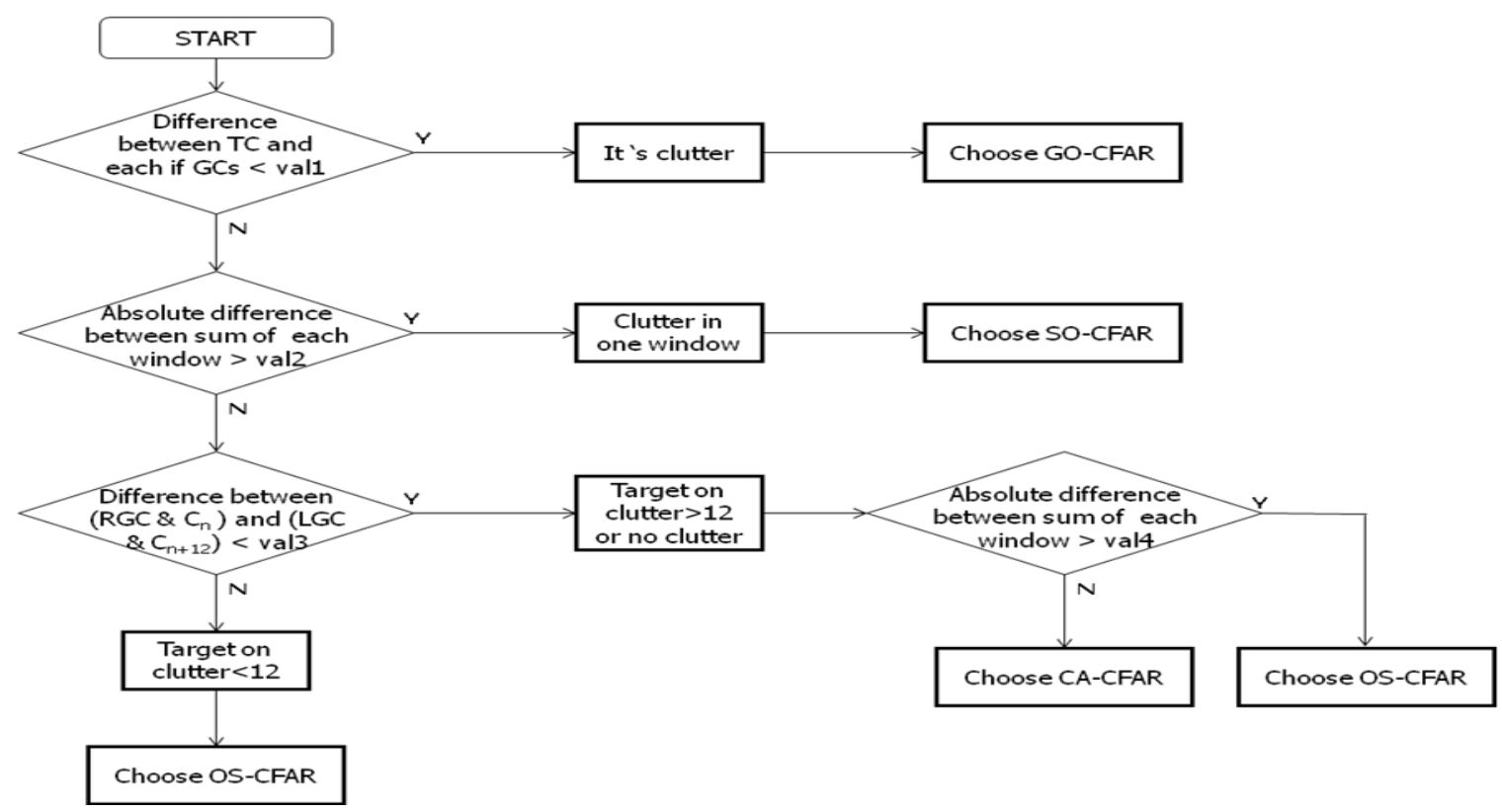

Fig. 4 Flow Chart of the threshold selection algorithm in the proposed INT-CFAR processor 


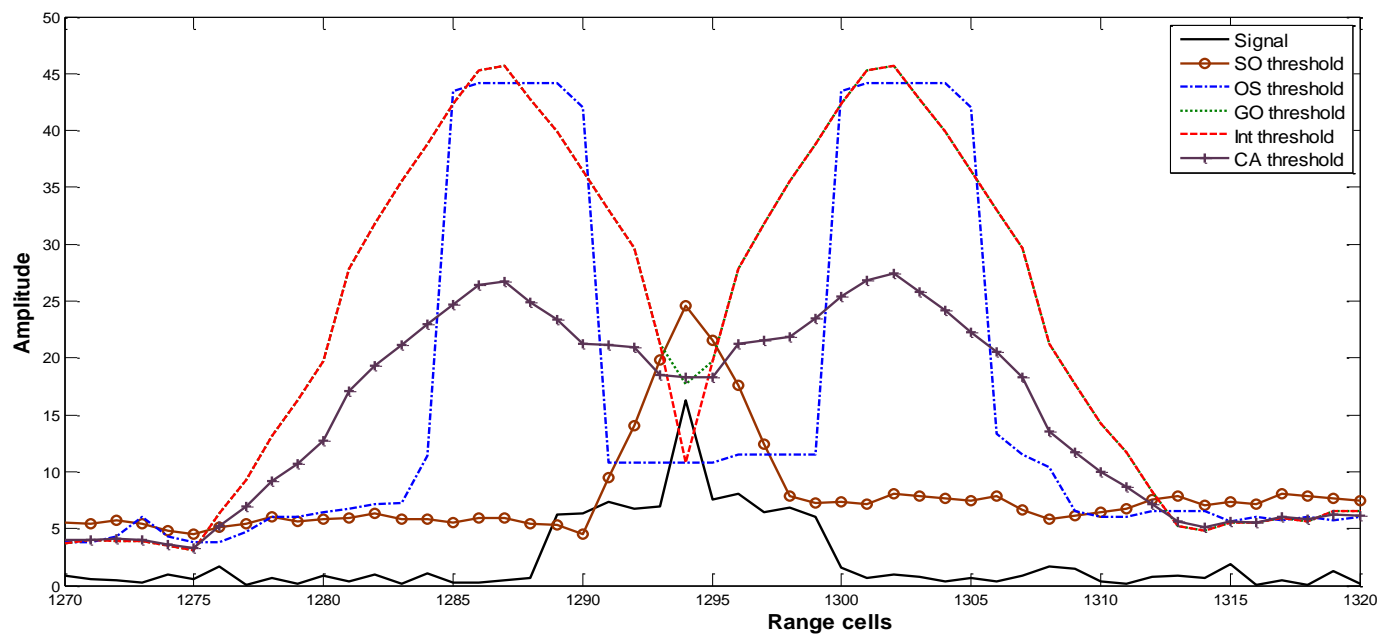

Fig. 5. Different CFAR thresholds in case of a target within a clutter region

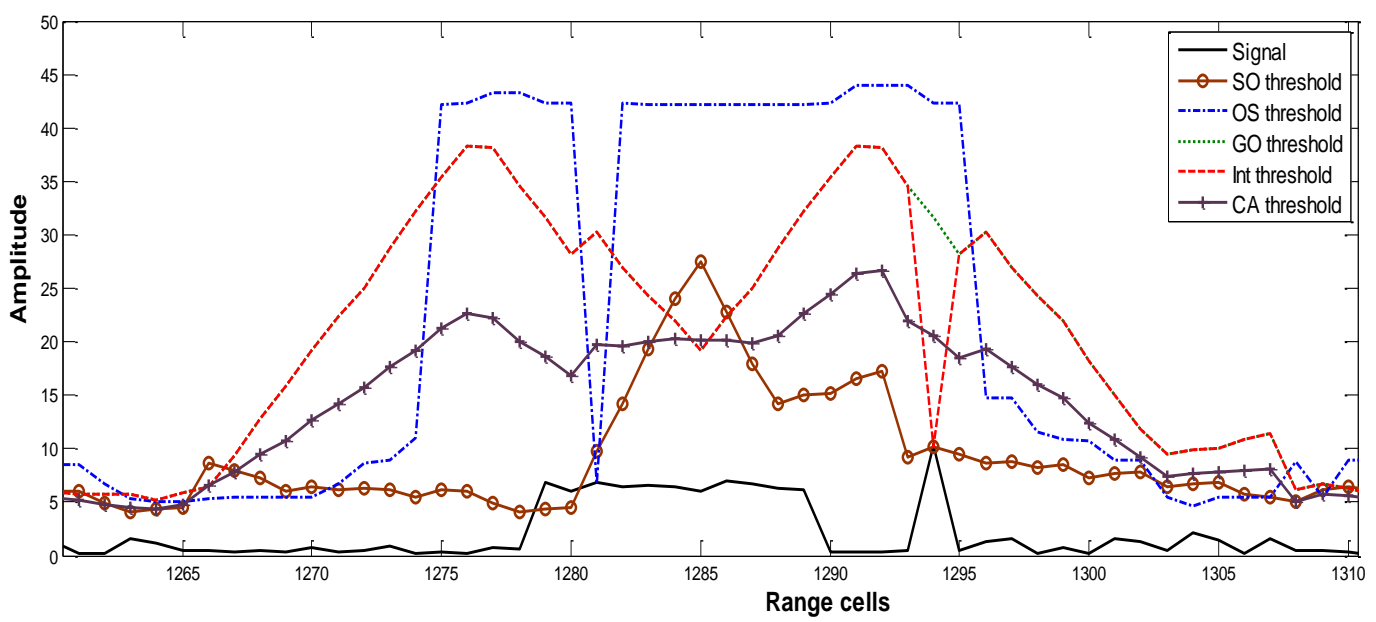

Fig. 6. Different CFAR thresholds in case of a target with clutter in one of the reference Windows

\subsection{Performance Evaluation}

The performance of the proposed INT-CFAR is compared against the CA, GO, SO, OS, and OSGO-CFAR processors using Matlab package. The ROC is used as a performance measure at different target and clutter situations.

The simulation parameters are as follows:

- CFAR total window size is 24 cells plus two guard cells and the test cell

- The target is represented by one sample in one cell.

- The designed probability of false alarm is $10^{-5}$

- The added noise is assumed to be White Gaussian Noise with zero mean and unity variance

- The added clutter is assumed to be with a Rayleigh probability density function

- The results are obtained for 100,000 trials 
Different target and clutter situations are as follows:

- The first case simulates the existence of one target in a homogeneous background

- The second case simulates the existence of one target in the TC and another interfering target in one of the reference windows.

- The third case simulates the existence of one target in the TC and clutter in one of the reference windows.

- The fourth case simulates the existence of a target within a clutter region.

For the first simulated case (existence of one target in a homogeneous background), figure 7 shows that the CA-CFAR introduces the best $\mathrm{P}_{\mathrm{d}}$, and the proposed INT-CFAR comes slightly lower.

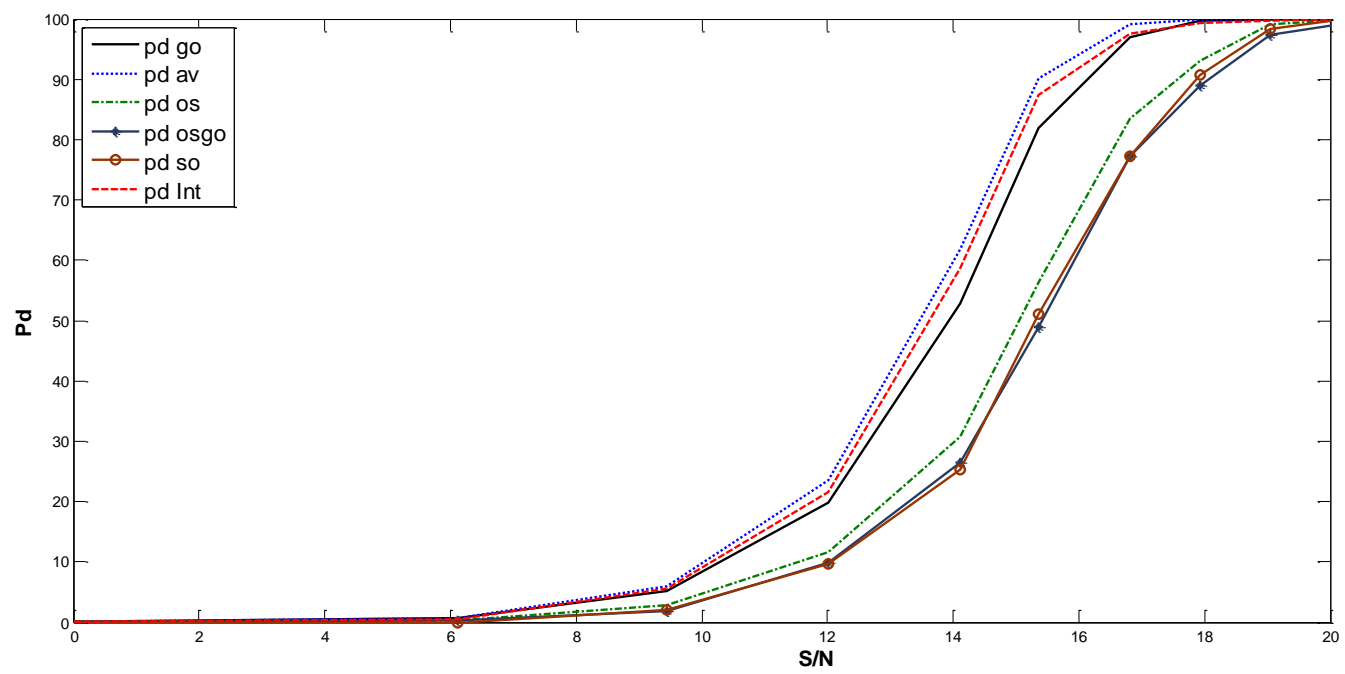

Fig. 7 The ROC curves for the first simulated case (existence of one target in a homogeneous background)

For the second case, (existence of one target in the TC and another interfering target in one of the reference windows), figure 8 shows that the proposed INT-CFAR introduces a nearly similar detection performance like that of the OS-CFAR processor outperforming the other CFAR processors.

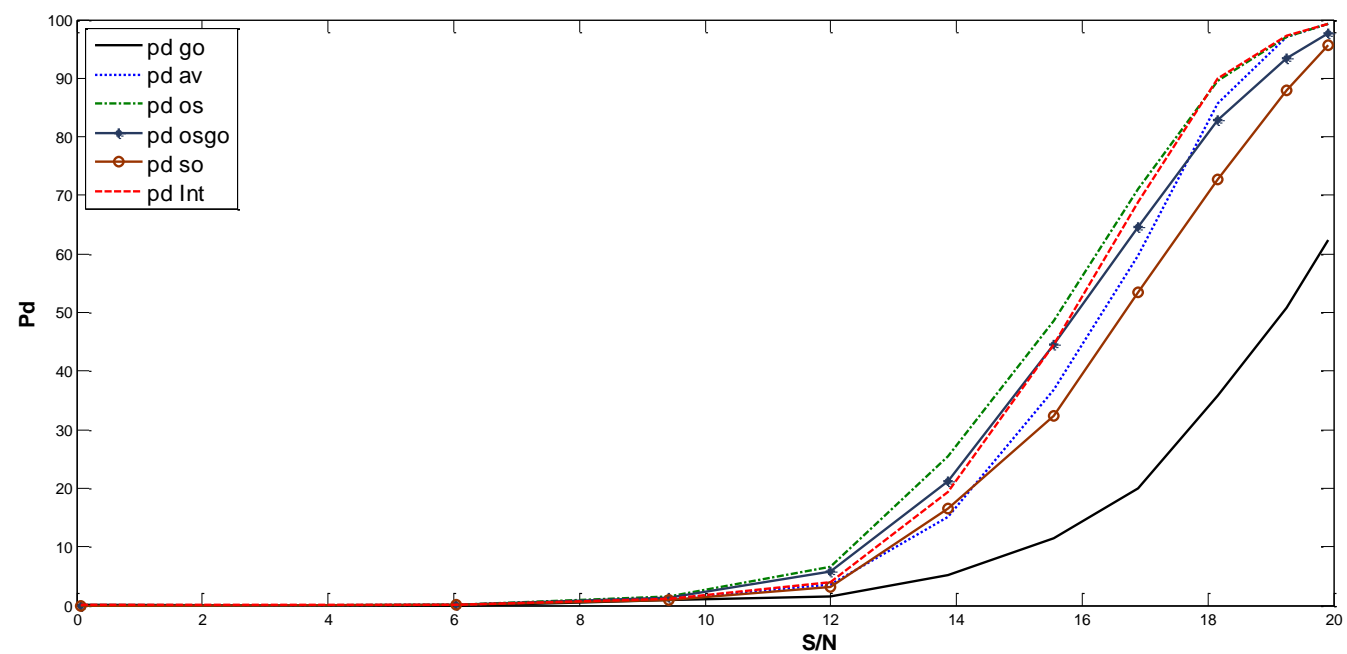

Fig. 8 The ROC curves for the second simulated case (existence of one target in the TC and another interfering target in one of the reference windows) 
Figure 9 shows the ROC curves for the third case (the existence of one target in the TC and clutter in one of reference windows). It's clear that the proposed INT-CFAR gives the best performance identical to that of the SO-CFAR processor.

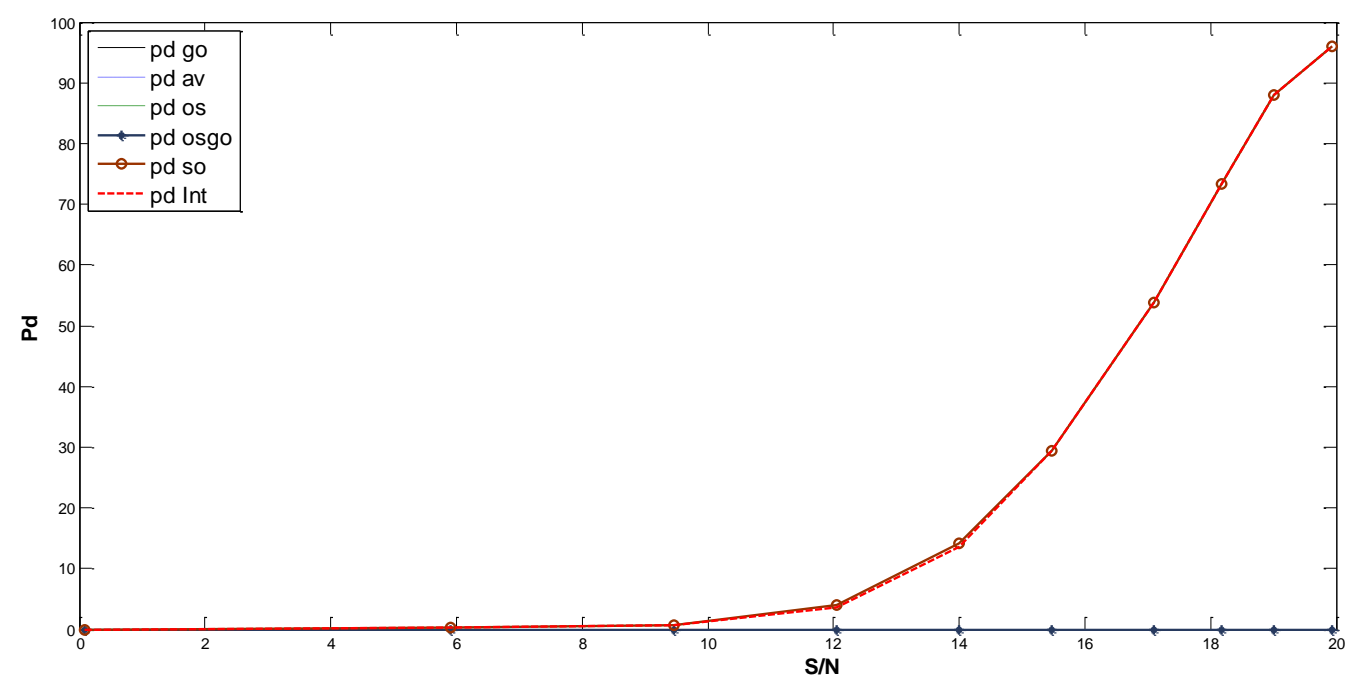

Fig. 9 The ROC curves for the third simulated case (existence of one target in the TC and clutter in one of the reference windows)

Concerning the fourth case, which is the existence of a target within the clutter region, figure 10 shows that the proposed INT-CFAR processor also gives almost the best detection performance like that of the OS-CFAR processor.

Results obtained from figures 7 through 10 showed that the proposed INT-CFAR processor gives almost the same best performance for all the discussed cases. The reason for the slight deviation between the proposed INT-CFAR processor and the best processor in each case may come from inaccurate choice of the selected variables (val1, val2, val3, and val4). These values were chosen according to a limited number of simulation trials.

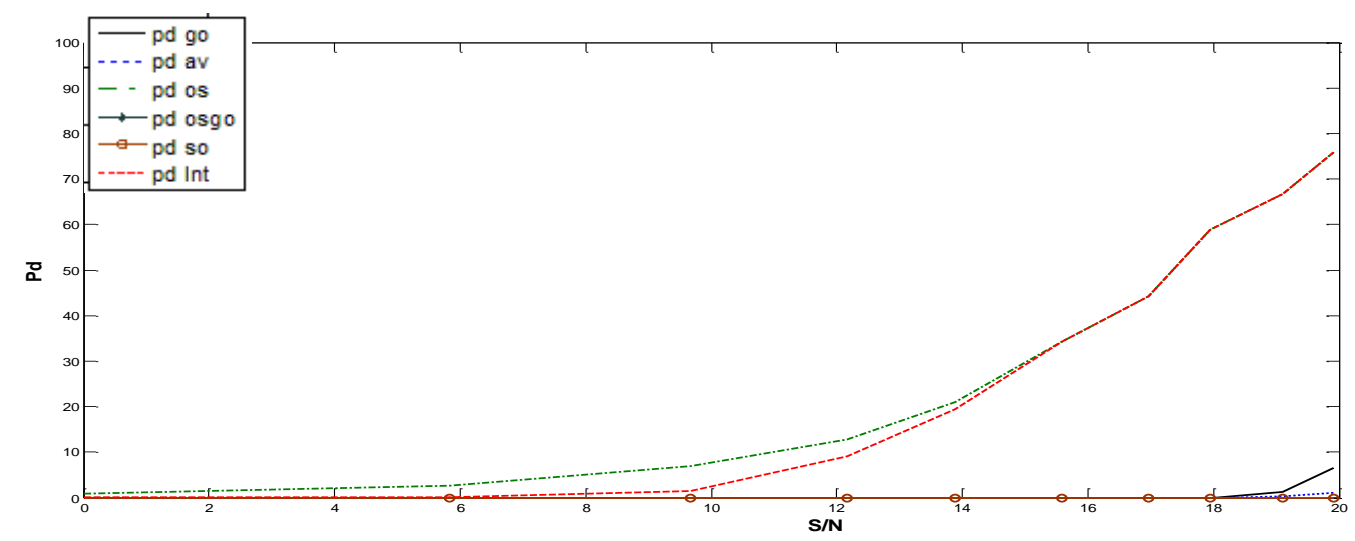

Fig. 10 The ROC curves for the fourth simulated case (existence of a target within a clutter region)

Figure 11 shows the variation of the probability of false alarm at different range cells for the situation where a clutter transition exists. It's clear that the $\mathrm{P}_{\mathrm{fa}}$ for the proposed INT-CFAR processor is much smaller than other CFAR processors especially at clutter edges. Also the proposed INT-CFAR processor is found to give almost a constant $\mathrm{P}_{\mathrm{fa}}$ at different range cells outperforming other CFAR processors. 
Another test is performed to measure the dependence of the $\mathrm{P}_{\mathrm{fa}}$ on the clutter to noise ratios (CNRs). This dependency is shown in figure 12. It's clear that the proposed INT-CFAR processor gives the lowest $\mathrm{P}_{\mathrm{fa}}$ at different CNRs and the steadiest one.

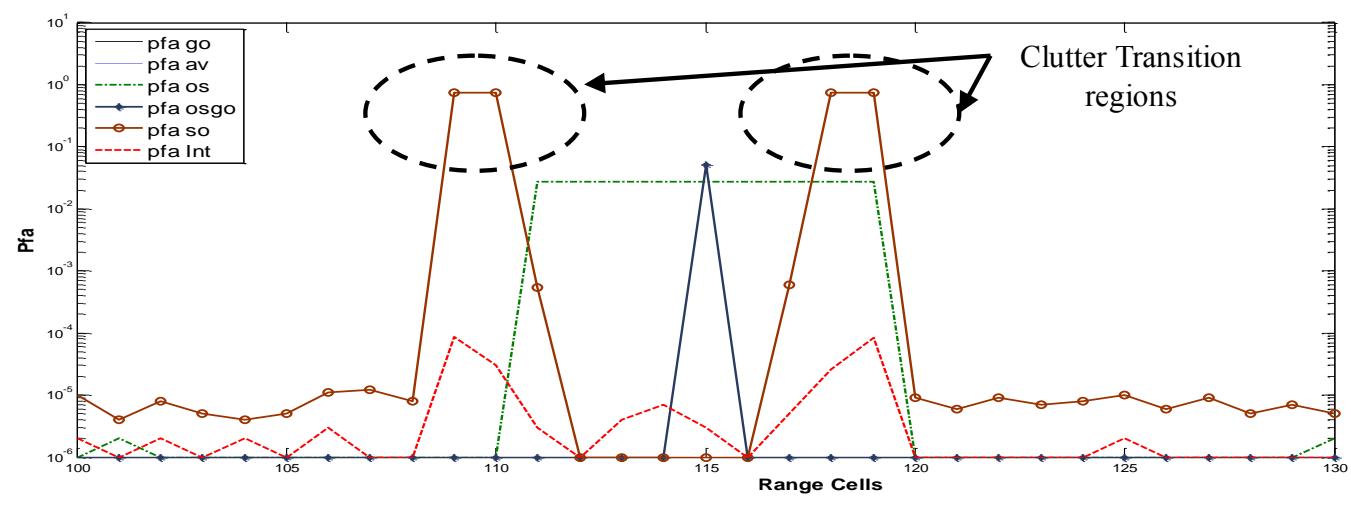

Fig. 11 Probability of false alarm at different range cells for different CFAR Processors

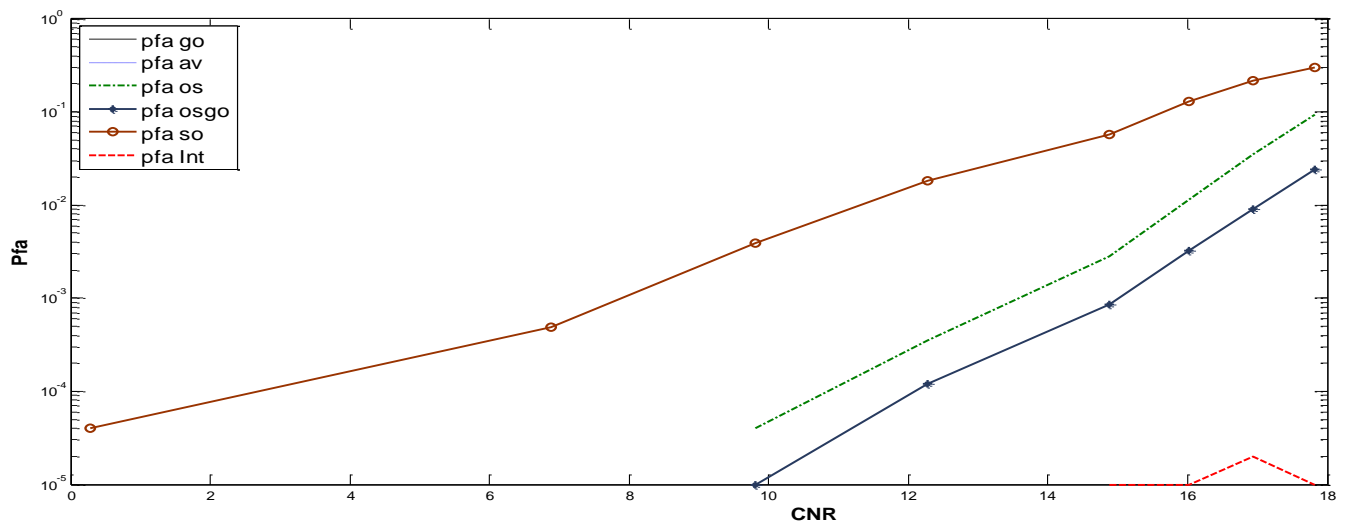

Fig. 12 Probability of false alarm at different range cells for different CFAR Processors

\section{Conclusion}

The theoretical aspects of different CFAR algorithms (CA, GO, SO, OS, and OSGO CFAR) are introduced. A new CFAR algorithm, designated as Intelligent (INT)-CFAR processor, is proposed. The main idea of the proposed CFAR is to select the optimum threshold value calculated by different CFAR processors, based on the information contained in the guard cells. The performance of the proposed INT-CFAR processor is evaluated and compared to other CFAR processors through the ROC curves at different target and clutter situations. The proposed INT-CFAR was found to be an optimum CFAR processor for all the studied cases. It gives the best detection performance with the minimum false alarm probability. The obtained results were based on computer simulations to validate the superiority of the proposed processor. 


\section{References}

[1] Rohling.H. (1983), Radar CFAR Thresholding in clutter and multiple target situations. IEEE, Transactions on Aerospace and Electronic Systems, pp 608-621, AES-19 (July 1983).

[2] Finn, H. M., and Johnson, R. S. (1968) Adaptive detection mode with threshold control as a function of spatially sampled clutter-level estimates. RCA Review, 29 (Sept. 1\%8), 414-464.

[3] Scharf, L. L., and Lytle, D. W. (1971) Signal detection in Gaussian noise of unknown level: An invariance application. IEEE Transactions on Information theory, IT-17 (July 1971), 404411.

[4] Hansen, V.G., Constant false alarm rate processing in search radars. In Proceedings of the IEEE International Radar Conference, London, pp. 325-332, (1973)

[5] Hansen, V.G. and Sawyers, J.H., Detestability loss due to greatest of selection in a cellaveraging CFAR, IEEE Transaction on Aerospace and Electronic Systems, pp 115-118, AES-16 (Jan. 1980).

[6] Moore. J.D., and Lawresnce. N.B , Comparison of two CFAR methods used with square law detection of Swerling I targets, In Proceeding of the IEEE International Radar Conference, pp 403-409, (1980).

[7] Weiss, M. , Analysis of some modified cell-averaging CFAR processors in multiple target situations, IEEE Transaction on Aerospace and Electronic Systems, pp 102133,AES-18 (Jan. 1982)

[8] Trunk, G. V., Range resolution of target using automatic detectors. , IEEE Transaction on Aerospace and Electronic Systems, pp 750-755, AES-14 (Sep. 1978)

[9] Gandhi P.P., Kassam S.A., "Analysis of CFAR Processors in Nonhomogeneous background," IEEE Transactions on Aerospace and Electronic Systems, vol. 24, no. 4, pp. 427-445, 1988.

[10] Roberto Perez-Andrade, Rene Cumplido, “A Versatile Hardware Architecture For A Cfar Detector Based On A Linear Insertion Sorter International Conference on Field Programmable Logic and Applications (FPL 2008), pp 467-470, 2008

[11] ALAN DI CENZO," Analysis of Some Modified Ordered Statistic CFAR, OSGO and OSSO CFAR", IEEE Transactions on Aerospace and Electronic Systems, pp 197-202, (1990). 